\title{
Design of a Backscatter-Free Waveguide Based on Photonic Topological Insulators
}

\author{
Mohammadreza Mehdipour ${ }^{\mathrm{a}}$, Vahid Ahmadi ${ }^{\mathrm{a}, \mathrm{b}, *}$, Reza Poursalehi $^{\mathrm{b}}$ \\ ${ }^{a}$ Department of Nanotechnology Engineering, Tarbiat Modares University, Tehran, Iran \\ bepartment of Electrical \& Computer Engineering, Tarbiat Modares University, Tehran, Iran \\ *Corresponding Email address: v_ahmadi@modares.ac.ir
}

Regular Paper: Received: Jun. 14, 2020, Revised: Jul. 23, 2020, Accepted: Aug. 6, 2020, Available Online: Aug. 7, 2020, DOI: 10.29252/ijop.14.1.85

\begin{abstract}
Topological Insulators are systems where the broken time reversal symmetry gives rise to protected edge modes that support backscatter-free and one-way propagation of electromagnetic waves by opening non-trivial bandgaps. In this study we investigate a oneway topologically protected waveguide in the frequency range of $f=6.0$ to $8.0 \mathrm{GHz}$. The time reversal symmetry is broken by an applied magnetic field in the $z$ direction. We show that the waveguide propagates the light in only one direction that can be controlled by the applied magnetic field and no backscattering is present in the waveguide which results in a near $100 \%$ transmission of light to the output. Furthermore, we investigate effect of the applied magnetic field on the topological properties of the system by considering the material dispersion of the rods. Our results show that 3 different frequency ranges will be supported by the edge modes at each given magnetic field. By increasing the magnitude of the applied magnetic field, a blue shift in the non-trivial bandgap is seen, where it can be used to tailor the modes for the waveguide.
\end{abstract}

KEYWORDS: Photonic Topological Insulators, Photonic Crystals, One-way Propagation, Edge Modes

\section{INTRODUCTION}

Topological photonics has been a promising field of research, where edge state modes exhibit in the system and provide one-way propagation of electromagnetic fields which are robust against impurities and defects and no backscattering is allowed [1-5]. Photonic band gaps appearing in photonic crystals (PCs) has inspired many applications in integrated optics. Namely, PCs made by magneto-optical materials are used in fabricating nonreciprocal optical circuits [6, 7]. Recently, backscatter-free gyromagnetic photonic crystal waveguides have drawn much attention due to their potential applications in circulators and switches. The modes, defined by edge states that have group velocities directed in only one direction are confined to the edge of the magneto-optical photonic crystal and can be controlled by an applied magnetic field. Hence, no backscattering exists in such systems due to the lack of back propagating modes. In order to achieve topological protected edge states time reversal symmetry is broken in the system by an applied magnetic field. In this paper we propose a waveguide and investigate the effect of the applied magnetic field on the topological edge states of the system.

\section{THEORY AND SIMUlation}

The basic structure is a 2D honeycomb photonic crystal with radius $0.3 a$, where $a$, is the lattice constant (Fig. 1(a)). In Fig. 1(a) each circle represents a rod with relative permittivity of $\varepsilon=15 \varepsilon_{0}$. When a DC magnetic field is applied along the axis of the rods, the field would induce the magnetic anisotropy of the ferromagnetic rods. After the material is fully magnetized, the magnetic permeability tensor $\mu$ is given by 
$\bar{\mu}=\left[\begin{array}{ccc}\mu_{r} & -i \mu_{k} & 0 \\ i \mu_{k} & \mu_{r} & 0 \\ 0 & 0 & 1\end{array}\right]$

where

$\mu_{r}=1+\frac{\omega_{m} \omega_{0}}{\omega_{0}^{2}-\omega^{2}}$

$\mu_{k}=\frac{\omega_{m} \omega}{\omega_{0}^{2}-\omega^{2}}$

The precession frequency is

$\omega_{0}=\gamma H_{0}$

where $\gamma=2.8 \times 10^{-3} \mathrm{C} / \mathrm{Kg}$ is the gyromagnetic ratio and $H_{0}$ is the applied magnetic field.

The dispersion relation of the structure is calculated using the conventional band theory of photonic crystals. In order to have one-way edge states, one must break the time reversal symmetry of the magneto optical photonic crystal modes which directly affects the topological properties of the bands of the system. This effect is generally defined by the Chern number, previously studied in quantum Hall effect extensively. The Chern number of the $\mathrm{n}$-th band is defined as

$$
C_{n}=\frac{1}{2 \pi i} \int_{B Z} d^{2} k\left(\frac{\partial A_{y}^{n n}}{\partial k_{x}}-\frac{\partial A_{y}^{n n}}{\partial k_{y}}\right)
$$

The integral in (5) is over the entire Brillouin zone and $A^{n n}$ is the Berry phase defined as

$$
A^{n n}(k)=\left\langle E_{n k}\left|\nabla_{k}\right| E_{n k}\right\rangle
$$

which is performed over the unit cell. Here $E_{n k}$ is the eigenvalue of the $n$-th band.

Each band has a Chern number which is shown to be always an integer, and if the band is time reversal symmetric the Chern number in Eq. (5) is always equal to zero. It is also possible to adiabatically tune the Hamiltonian by changing the permeability tensor of the system. In this case, the Chern number of the system can be tuned by the applied magnetic field. In fact, this change happens where the band is degenerate and by applying the magnetic field the degeneracy is lifted and discrete degenerate points show up, a bandgap opens and the Chern number of the band changes by $\Delta C$. Thus, we look for a time reversal symmetric band structure with a pair of photonic bands degenerate at a specific wave vector.

The band structure for the applied magnetic field $H=0.1 \mathrm{~T}$ around $k=0$ is depicted in Fig. 1(b) where a band gap is shaped by the broken time reversal symmetry. The solid lines represent the eigenvalues of the photon energy inside the photonic crystal structure where a gap is opened in frequency range $f=7.0$ to $f=7.3 \mathrm{GHz}$ at $k=0$. However, the dashed lines inside the gap represent the edge modes of the photons that have different Chern number of $\Delta C=1$. Hence giving rise to the topologically protected states that can propagate in one direction only, and are immune to backscattering.

(a)

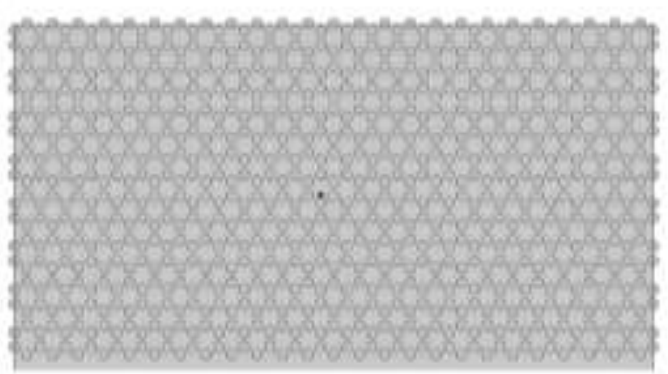

(b)

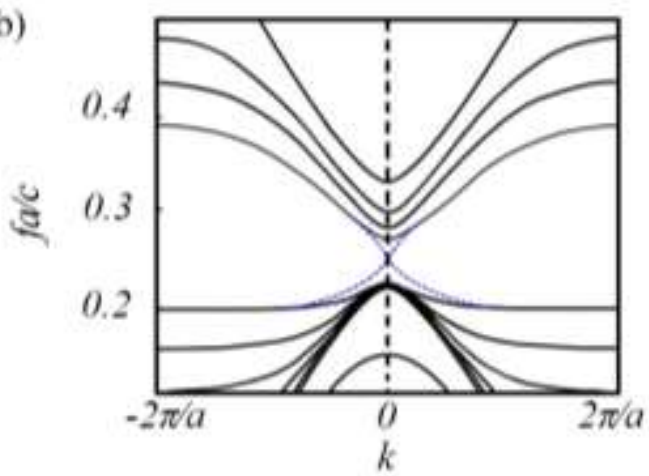

Fig. 1. (a) The honeycomb structure of ferrite rods is air with lattice constant a. (b) Dispersion relation of the honeycomb structure. Time reversal symmetry is broken in the band by applying a 
magnetic field, leading to one-way edge states inside the bandgap.

\section{RESULT AND DISCUSSION}

\section{A. Designing a waveguide}

In order to design a waveguide, we consider the structure depicted in Fig. 1(a) adjacent with another photonic crystal with the same geometry but the rods have a permeability tensor with mirrored off-diagonal elements with that of Eq. (1).

Figure 2 shows the structure of the waveguide where the blue (top) domain is the same as Fig. 1(a) and the red (bottom) domain has mirrored off-diagonal elements with respect to the blue (top) domain. The space created between the two domains acts as a waveguide for the frequency range inside the bandgap of the top and bottom photonic crystals and only edge modes will be allowed to propagate inside the waveguide, which are topologically protected and can only propagate in one direction only.

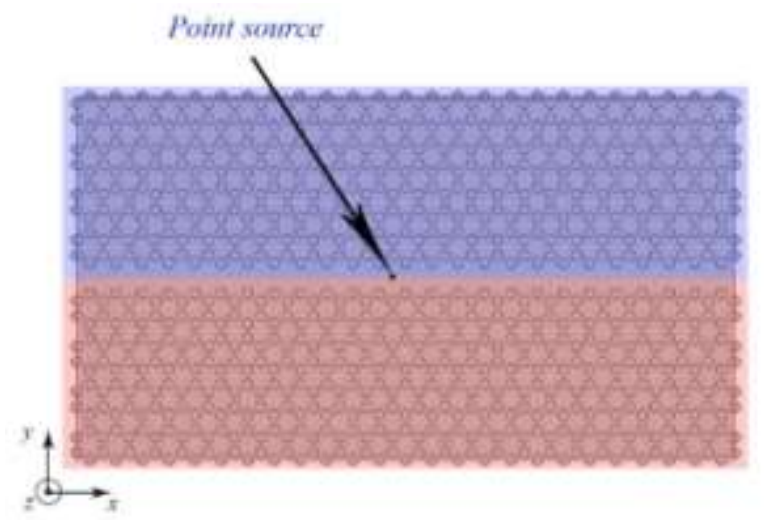

Fig. 2. Two domains with mirrored off-diagonal elements in their permeability tensor can form a one-directional waveguide

To confirm the one-way topologically protected edge modes we introduce a point source in the middle of the waveguide formed by two domains (Fig. (2)). The top domain only supports modes that can propagate around it clockwise and the bottom domain only supports modes that propagate around it in a counter clockwise direction. As shown in Fig. 3(a) it can be seen that frequencies with different Chern number only propagate to the left and no propagation of light is evident as there are no modes to support propagation to the right. Therefore, it is confirmed that a oneway propagating waveguide can be formed by closing in the two domains with mirrored magnetic permeability tensors. By changing the direction of the applied magnetic field to $z$, it can be seen that the propagating modes also change their direction (inset of Fig. 3(a)).

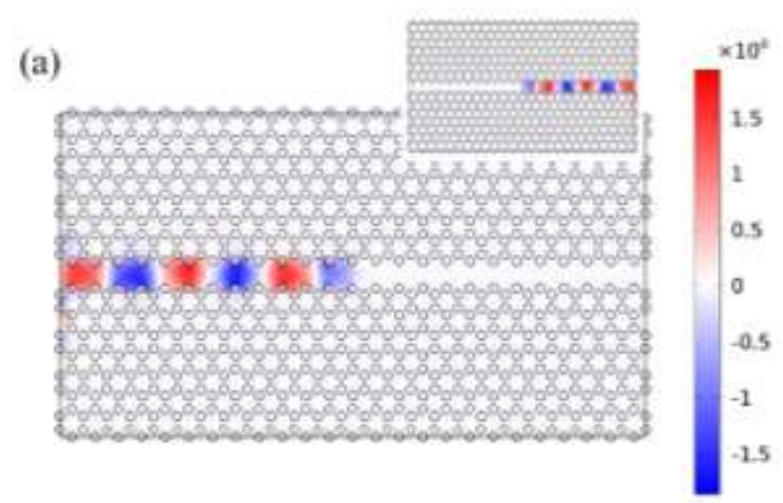

(b)

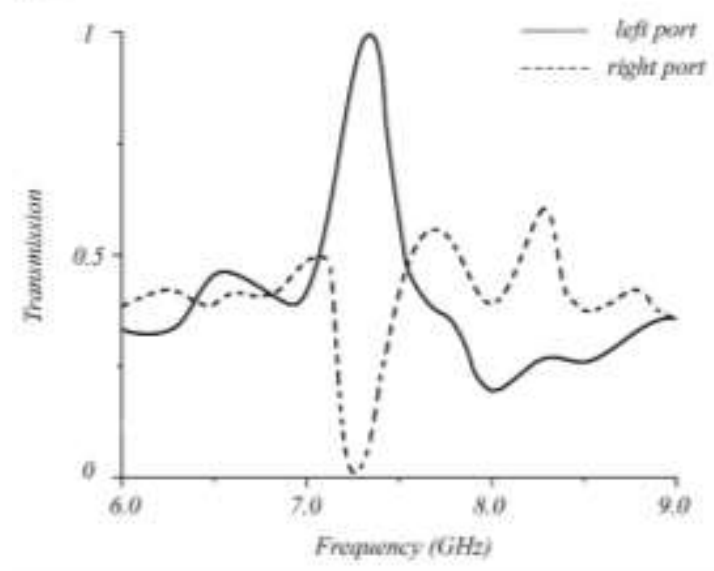

Fig. 3. (a) The electric field profile at $\mathrm{f}=7.25 \mathrm{GHz}$ shows that propagation of the electromagnetic field is only allowed to the left in the waveguide. The inset shows the direction of the propagating light can change by the direction of the applied magnetic field. (b) The transmission spectrum of the waveguide shows that at $\mathrm{f}=7.25 \mathrm{GHz}$ all the energy is propagated to the left port and zero energy is received at the right port.

Also, we calculate the energy received at the left and right port of the waveguide when a $H=0.1$ ( $\mathrm{T}$ ) magnetic field in the $\mathrm{z}$ direction is applied to the system (Fig. 3(b)). It can be 
clearly seen that at $f=7.25 \mathrm{GHz}$ a near $100 \%$ transmission of light is received at the left port where at the same frequency the energy received at the right port drops to zero. This can confirm that this is a novel design for oneway propagating waveguide where there are no back scatterings allowed in the desired frequency range. This unique feature can be promising in the on/off switches since the light output can be controlled by the direction of the applied magnetic field.

\section{B. Effect of applied magnetic field}

To study further we consider the effect of the applied magnetic field on the topological properties of the system. Since the permeability tensor of the rods changes with the magnitude of the applied magnetic fields as given by Eq. (4), the effect of material dispersion cannot be neglected. Therefore, we consider the permeability tensor of the rods as dispersive and changed with respect to the applied magnetic field.

We change the applied magnetic field in the $z$ direction from zero to $0.1 \mathrm{~T}$, and study the change in the band structure of the system. Figure (4) shows the non-trivial bandgap map of the system when we change the applied magnetic field. We only show the non-trivial bandgap of the system as the topologically protected edge states exists and support oneway propagation of the electromagnetic field. It is interesting to point out that for every applied magnetic field, 3 non-trivial bandgaps open and topological edge modes appear. By increasing the applied magnetic field, the bandgaps are shifted to higher frequencies. In other words, increasing the magnetic field induces a blue-shift in the topologically protected edge modes of the waveguide. At $H=0.1 \mathrm{~T}$ the top and bottom non-trivial bandgaps in Fig. 4 are closed and only one bandgap is available at frequency range $f=7.04$ $\mathrm{GHz}$ to $f=7.32 \mathrm{GHz}$ and for higher amplitude magnetic fields no bandgaps can be seen, therefore no edge states are present.

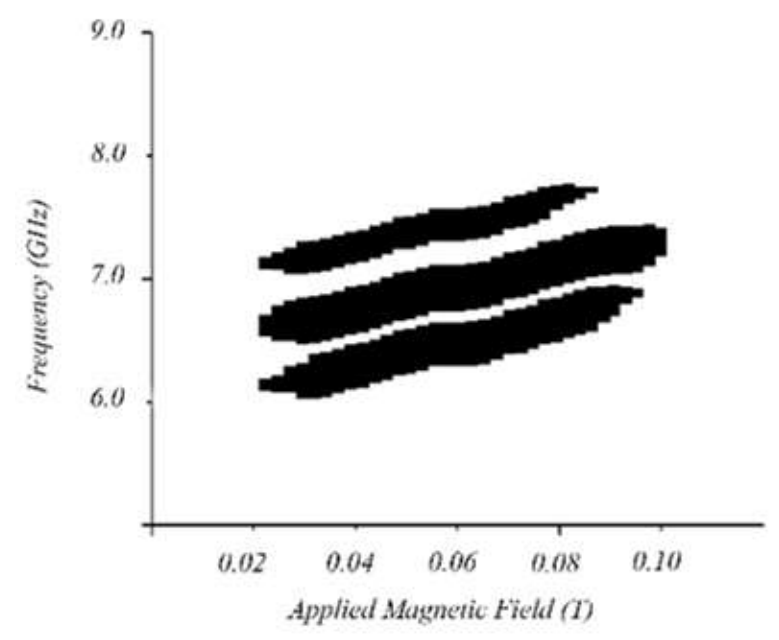

Fig. 4. The bandgap map of the proposed waveguide shows three ranges of non-trivial bandgap for every applied magnetic field. The top and bottom gaps close by increasing the applied magnetic field to $H=0.1 T$.

It is evident that we can control the topological properties of the system by changing the applied magnetic field. Also, it is possible to use this waveguide as an on/off switch at frequency range $f=7.04 \mathrm{GHz}$ to $f=7.32 \mathrm{GHz}$. Where the power of the electromagnetic wave is delivered near $100 \%$ and no backscattering is allowed, plus no power is received at the right port. By integrating the multiple switches, optical logic gates can be designed which are tunable by the applied magnetic field. Also, one of the most promising applications of such systems can be considered as cloaking, as no back scattering of light is allowed in a specific frequency range and the propagation direction of the incident electromagnetic waves can be controlled by a suitable design.

\section{CONCLUSION}

We have proposed a waveguide based on photonic topological insulators by putting two different domains of honeycomb photonic crystals with mirrored magnetic permeability tensors near each other. By breaking the time reversal symmetry in the system, protected edge modes appear that only support propagation of light in one direction in the waveguide. It can be seen that for an applied magnetic field of $H=0.1 \mathrm{~T}$ protected edge 
modes allow propagation of light in the frequency range of $f=7.04 \mathrm{GHz}$ to $f=7.32 \mathrm{GHz}$ to one side only with no backscattering in the system. In this frequency range all the energy is delivered to the left port and zero energy reaches the right port which confirms the oneway edge modes of the system.

Furthermore, the effect of applied magnetic field was studied by considering the material dispersion of the rods. In the band-gap map of the system, 3 different non-trivial gaps open for applied magnetic fields higher than $H=0.02 \mathrm{~T}$. By increasing the magnitude of the applied magnetic field, the gaps also shift to higher frequencies. At $H=0.1 \mathrm{~T}$ the top and bottom non-trivial band gaps close and only one gap is present in frequency range $f=7.04$ $\mathrm{GHz}$ to $f=7.32 \mathrm{GHz}$. By changing the direction of the applied magnetic field, the direction of the propagating modes can be controlled which can be used as an on/off switch either. In general, the ability to control the light perfectly can give rise to infinite applications in future optical integrated circuits.

\section{ACKNOWLEDGEMENT}

This work was financially supported by Iran National Science Foundation (INSF). The authors would like to acknowledge the financial support received from Tarbiat Modares University, through grant \#IG-39703.

\section{REFERENCES}

[1] C.L. Kane and E.J. Mele, "Z2 topological order and quantum spin hall effect," Phys. Rev. Lett. Vol. 95, pp. 146802-146806, 2005.

[2] N. H. Lindner, G. Refael, and V. Galitski, "Floquet Topological insulator in semiconductor quantum wells," Nat. Phys. Vol. 7, pp. 490-495, 2011.

[3] T. Ma, A. B. Khanikaev, S. H. Mousavi, and G. Shvets, "Guiding electromagnetic waves around sharp corners: Topologically protected photonic transport in metawaveguides," Phys. Rev. Lett. Vol. 114, pp. 12740 (1-7), 2015.
[4] X.-L. Qi and Sh.-Ch. Zhang, "Topological insulators and superconductors," Rev. Modern Phys. Vol. 83, pp. 1057-1110, 2011.

[5] S. A. Skirlo, L. Lu, and M. Soljačić, "Multimode one-way waveguides of large chern numbers," Phys. Rev. Lett. Vol. 113, pp. 113904-113909, 2014.

[6] Z. Wang and S. Fan, "Optical circulators is two-dimensional magneto-optical photonic crystals," Opt. Lett. Vol. 30, pp. 1989-1991, 2005.

[7] P. Wang, L. Lu, and K. Bertoldi, "Topological phononic crystals with one-way elastic edge waves," Phys. Rev. Lett. Vol. 115, pp. 104302-104307, 2015.

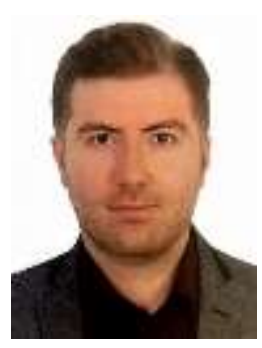

Mohammadreza Mehdipour received the B.Sc. degree in applied physics from Department of Physics, University of Sistan and Balouchestan, Zahedan, in 2011 and the M. Sc. in Nanotechnology from Department of Nanomaterials Engineering, Tarbiat Modares University in 2014, where he is currently a $\mathrm{Ph}$. D. student. His research interests are optoelectronic photonic crystal devices.

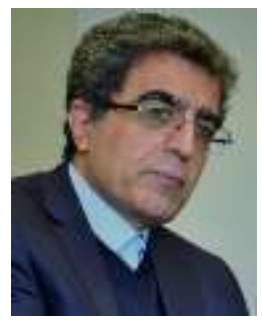

Vahid Ahmadi (M'86-SM'09) received the $\mathrm{Ph} . \mathrm{D}$. degree in electronic engineering from the Kyoto University, Japan, in 1994. He was the Head of the Semiconductor Department of Laser Research Centre, Tehran, from 1994 to 2006. He was the Head of the Electrical Engineering Department, Tarbiat Modares 
University (TMU), from 2006 to 2008 . He is currently a Professor in electronic engineering with TMU, Tehran, Iran. His current research interests include nano-optoelectronic devices, nanophotonic, plasmonic, and biophotonic devices, nonlinear optics and slow light, optical quantum devices, tunable lasers and detectors, optical modulators, amplifiers and switches, micro-ring-based devices, photonic crystal and metamaterial-based devices, organic-based light sources and sensors, Perovskite-based solar cells and novel devices, photonic topological insulator-based devices, and graphene and 2D materials-based photonic devices. He is the member of the FoundersBoard of Optics and Photonics Society of Iran; the editorial board of International Journal of Information and Communication Technology Research (IJICTR). He was the Technical and Scientific Chair of 13th Iranian Conf. on Optics and Photonics, in 2007, and the General Chair of the 16th Iranian Conference on Electrical Engineering (ICEE2008), in 2008. $\mathrm{He}$ is currently the Chair of the Optoelectronics and Photonics Research Group and the Head Nano-Optoelectronics
Laboratory, TMU. He is also the Editor-inChief of Modares Journal of Electrical Engineering.

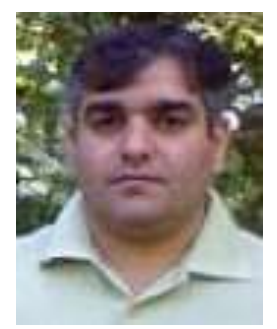

Reza Poursalehi earned his B.Sc, M.Sc, and $\mathrm{PhD}$ degrees all from Department of Physics, Sharif University of Technology. $\mathrm{He}$ is currently an associate professor of Nanomaterials at department of Materials Engineering, Tarbiat Modares University. His main research interests are in the field of nanomaterials synthesis, processing and applications especially, laser processing of nanomaterials, oxide semiconductors, arc discharge synthesis and processing of nanomaterials, photo-catalysts, water splitting and optical nanomaterials. 